\title{
Trehalose treatment suppresses inflammation, oxidative stress, and vasospasm induced by experimental subarachnoid hemorrhage
}

Ryosuke Echigo ${ }^{1 \dagger}$, Nobuyuki Shimohata ${ }^{2,3^{*}+}$, Kensuke Karatsu', Fumiko Yano ${ }^{3}$, Yuko Kayasuga-Kariya ${ }^{4}$, Ayano Fujisawa ${ }^{4}$, Takayo Ohto ${ }^{5}$, Yoshihiro Kita ${ }^{5}$, Motonao Nakamura ${ }^{5}$, Shigeki Suzuki ${ }^{2}$, Manabu Mochizuki ${ }^{1}$, Takao Shimizu ${ }^{5}$, Ung-il Chung ${ }^{4}$ and Nobuo Sasaki ${ }^{1}$

\begin{abstract}
Background: Subarachnoid hemorrhage $(\mathrm{SAH})$ frequently results in several complications, including cerebral vasospasm, associated with high mortality. Although cerebral vasospasm is a major cause of brain damages after $\mathrm{SAH}$, other factors such as inflammatory responses and oxidative stress also contribute to high mortality after SAH. Trehalose is a non-reducing disaccharide in which two glucose units are linked by a,a-1,1-glycosidic bond, and has been shown to induce tolerance to a variety of stressors in numerous organisms. In the present study, we investigated the effect of trehalose on cerebral vasospasm, inflammatory responses, and oxidative stress induced by blood in vitro and in vivo.

Methods: Enzyme immunoassay for eicosanoids, pro-inflammatory cytokines, and endothelin-1, and western blotting analysis for cyclooxygenase-2, inducible nitric oxide synthase, and inhibitor of NF-kB were examined in macrophage-like cells treated with hemolysate. After treatment with hemolysate and hydrogen peroxide, the levels of lipid peroxide and amounts of arachidonic acid release were also analyzed. Three hours after the onset of experimental SAH, 18 Japanese White rabbits received an injection of saline, trehalose, or maltose into the cisterna magna. Angiographic and histological analyses of the basilar arteries were performed. In a separate study, the femoral arteries from 60 rats were exposed to fresh autologous blood. At 1, 3, 5, 7, 10, and 20 days after treatment, cryosections prepared from the femoral arteries were histologically analyzed.
\end{abstract}

Results: When cells were treated with hemolysate, trehalose inhibited the production of several inflammatory mediators and degradation of the inhibitor of NF-KB and also suppressed the lipid peroxidation, the reactive oxygen species-induced arachidonic acid release in vitro. In the rabbit model, trehalose produced an inhibitory effect on vasospasm after the onset of experimental SAH, while maltose had only a moderate effect. When the rat femoral arteries exposed to blood were investigated for 20 days, histological analysis revealed that trehalose suppressed vasospasm, inflammatory response, and lipid peroxidation.

Conclusions: These data suggest that trehalose has suppressive effects on several pathological events after SAH, including vasospasm, inflammatory responses, and lipid peroxidation. Trehalose may be a new therapeutic approach for treatment of complications after SAH.

Keywords: Trehalose, Subarachnoid hemorrhage, Cerebral vasospasm, Inflammatory response, Oxidative stress

\footnotetext{
* Correspondence: shimohata@cnbi.t.u-tokyo.ac.jp

${ }^{\dagger}$ Equal contributors

${ }^{2}$ NEXT21 K.K., 3-38-1 Hongo, Bunkyo-ku, Tokyo 113-0033, Japan

${ }^{3}$ Center for Disease Biology and Integrative Medicine, Faculty of Medicine,

The University of Tokyo, 7-3-1 Hongo, Bunkyo-ku, Tokyo 113-0033, Japan

Full list of author information is available at the end of the article
} 


\section{Background}

Aneurysmal subarachnoid hemorrhage (SAH) is a serious condition which often leads to high mortality and morbidity [1]. Ischemic injury due to cerebral vasospasm is a major cause of brain damage after SAH. However, cerebral vasospasm is only considered one of the underlying causes of SAH, and does not represent all of the associated clinical manifestations. A recent study also demonstrated that recovery of vasoconstriction by an endothelin antagonist did not necessarily contribute to improved clinical outcome [2], and additional mechanisms of secondary injury besides vasospasm have been suggested [3].

$\mathrm{SAH}$ occurs after the rupture of an aneurysm on the cerebral artery wall. Erythrocyte cytosol is released into the subarachnoid space through hemolysis of erythrocytes. Oxyhemoglobin or a related high molecular weight compound in erythrocyte cytosol can elicit a wide range of stress responses, including activation of inflammatory responses and production of inflammatory cytokines [4]. These actions are partially regulated by the NF- $\mathrm{kB}$ signaling pathway. The NF-kB signaling pathway is essential for host defense and inflammatory responses to extracellular stimuli, but also has been associated with production of inflammatory mediators after SAH [5]. It is also known that the production of a class of arachidonic acid-derived eicosanoids is enhanced in patients with SAH [6]. In addition, oxidative stress, including lipid peroxidation, can occur following SAH due to excessive free radicals generated by oxyhemoglobin and enzymatic reactions, and plays important roles in the pathogenesis of acute brain injury, development of vasospasm, and breakdown of the blood brain barrier [7]. In support, overexpression of antioxidant enzyme has been shown to attenuate early brain injury after SAH [8].

A number of human and animal studies have been performed on irrigation of the subarachnoid space for the removal of blood clots, percutaneous transluminal angioplasty, anti-inflammatory agents including nonsteroidal anti-inflammatory drugs and glucocorticoids, and antioxidants including radical scavengers and lipid peroxidation inhibitors $[4,9,10]$. However, there are still no definitive treatments for complications including vasospasm after $\mathrm{SAH}$. As the underlying causes of the complications following SAH are multifactorial, effective treatment will likely require a combination of approaches including clearance of blood and the use of anti-oxidant reagents and anti-inflammatory reagents, or a reagent with pleiotropic effects.

Trehalose is a non-reducing disaccharide in which two glucose units are linked by an $\alpha, \alpha-1,1$-glycosidic bond. Trehalose has multiple functions that distinguish it from other common disaccharides, including a protective action against stressors such as desiccation, reactive oxygen species (ROS), and cold [11,12]. In addition, recent reports showed that trehalose could prevent inflammatory responses induced by endotoxic shock in vivo and in vitro [13,14]. As such, trehalose is considered a potentially powerful therapeutic agent for various diseases, involving oxidative stress, desiccating conditions, and chronic inflammation. Trehalose is also considered to have a high safety profile as a trehalose-containing organ preservation solution is used in clinical lung transplantation [15]. Furthermore, clinical application of trehalose has been attempted for the cryopreservation of platelets, dry eye syndrome, and oral dryness caused by dental treatment [16-18].

In the present study, we examined whether trehalose could suppress oxidative stress, inflammatory responses, and cerebral vasospasm after SAH.

\section{Methods}

\section{Materials, animals and cell culture}

Trehalose and maltose were purchased from Wako (Osaka, Japan). The percentage of trehalose in solution using the in vitro and in vivo experiments was selected based on our preliminary data (data not shown).

Lipopolysaccharide (LPS) (from Escherichia coli O111: B4) was from Sigma (St. Louis, MO, USA), and anisomycin (from Streptomyces griseolus) was from Calbiochem (San Diego, CA, USA).

Male Japanese White rabbits, male Wistar rats, and male Sprague-Dawley (SD) rats were purchased from Japan SLC (Hamamatsu, Japan). The design of the animal study was approved by the Animal Care Committee of the Graduate School of Agricultural and Life Sciences, The University of Tokyo.

We investigated the effect of trehalose on bloodinduced inflammatory responses in macrophage cells and human umbilical vein endothelial cells (HUVECs). These cells play important roles in inflammation after SAH [19-22]. The hemolysate (see below) was used for in vitro studies on blood-induced inflammation. The murine macrophage cell line RAW 264.7 was obtained from the RIKEN Cell Bank (Ibaraki, Japan). RAW 264.7 cells were cultured in Dulbecco's Modified Eagle's Medium (DMEM) (Sigma) supplemented with 10\% (v/v) fetal bovine serum (FBS) (Gibco, Carlsbad, CA, USA) and $1 \%(\mathrm{v} / \mathrm{v})$ penicillin and streptomycin (Sigma). HUVECs were obtained from Lonza (Basel, Switzerland). HUVECs were grown in endothelial basal medium supplemented with growth factors and FBS (EBM-2; Lonza).

\section{Preparation of hemolysate}

Hemolysates from Wistar rats (weighing approximately $250 \mathrm{~g}$ ) were prepared according to a previously described method [23], with slight modifications. The erythrocyte fraction was washed three times with saline, and then erythrocytes were divided into three parts and suspended in either saline, $10 \%(\mathrm{w} / \mathrm{v})$ trehalose (final concentration, 
7.5\%), or $10 \%(\mathrm{w} / \mathrm{v})$ maltose (final concentration, $7.5 \%$ ), and then gently sonicated for $30 \mathrm{~s}$. After centrifugation, the supernatants were collected as erythrocyte hemolysates and stored at $-80^{\circ} \mathrm{C}$ until use. Two absorbance peaks at 540 and $576 \mathrm{~nm}$ were spectrophotometrically measured in hemolysate samples, confirming the presence of oxyhemoglobin.

\section{Enzyme immunoassay for eicosanoids, pro-inflammatory cytokines, and endothelin-1}

RAW 264.7 cells or HUVECs were incubated with or without $10 \%$ hemolysate in the medium mixed with an equal part of saline, $10 \%$ trehalose, or $10 \%$ maltose for $8 \mathrm{~h}$ or $18 \mathrm{~h}$, and then washed three times with fresh medium. After incubation for $1 \mathrm{~h}$, the culture media were collected and centrifuged at $15,000 \mathrm{rpm}$ for $10 \mathrm{~min}$. The supernatants were collected for tests. The supernatant concentrations of prostaglandin $\mathrm{E}_{2}\left(\mathrm{PGE}_{2}\right)$, cysteinyl leukotriene, human endothelin-1, tumor necrosis factor- $\alpha$ (TNF- $\alpha$ ), interleukin-6 (IL-6), interleukin-1 $\alpha$ (IL-1 $\alpha)$, and interleukin-1 $\beta$ (IL-1 $\beta$ ) were measured using enzyme immunoassay (EIA) kits (the first two with kits from Cayman, Ann Arbor, MI, USA; the remainder from R\&D Systems, Minneapolis, MN, USA) according to the manufacturer's instructions.

\section{Arachidonic acid release assay}

RAW 264.7 cells were pre-incubated for $24 \mathrm{~h}$ in basal medium containing $1 \mu \mathrm{Ci}\left[{ }^{3} \mathrm{H}\right]$-arachidonic acid/ml (PerkinElmer Life Sciences, Boston, MA, USA). The cells were washed three times with Tyrode solution containing $10 \mathrm{mM}$ HEPES- $\mathrm{NaOH}$ (pH 7.4) and $0.1 \%$ fatty acid-free bovine serum albumin (Sigma) (wash buffer) and incubated at $37^{\circ} \mathrm{C}$ for $3 \mathrm{~h}$ in wash buffer with $10 \%$ hemolysates, $1.5 \mathrm{mM} \mathrm{H}_{2} \mathrm{O}_{2}$, or the corresponding vehicle. At the end of the $3 \mathrm{~h}$ incubation period, the incubation medium was removed and centrifuged at 10,000 rpm. The supernatants were collected for tests. To determine the radioactivity of released arachidonic acid, aliquots of the supernatants were placed in scintillation vials containing scintillation fluid, and radioactivity was measured using a liquid scintillation counter LS6500 (Beckman Coulter, Fullerton, CA, USA). To determine the radioactivity of incorporated arachidonic acid, the cells that remained attached to the plates were lysed using $2 \% \mathrm{NP}$ 40 substitute solution, and the cell lysate was utilized for scintillation counting. The quantity of phospholipase $\mathrm{A}_{2}$ $\left(\mathrm{PLA}_{2}\right)$ activity was shown as the total radioactivity released per unit of incorporated radioactivity.

Western blotting analysis for cyclooxygenase-2, inducible nitric oxide synthase, and IKB-a

RAW 264.7 cells were incubated with or without $10 \%$ hemolysate, $20 \mathrm{ng} / \mathrm{ml} \mathrm{LPS}$, or $10 \mu \mathrm{M}$ anisomycin in medium mixed with an equal part of saline or 10\% trehalose for $8 \mathrm{~h}$ or at the time points indicated in figure legends. After washing with ice-cold PBS, cells were collected and lysed using TNE buffer $(10 \mathrm{mM}$ Tris- $\mathrm{HCl} \mathrm{pH}$ 7.8, $150 \mathrm{mM} \mathrm{NaCl}, 1 \mathrm{mM}$ EDTA, and 1\% NP-40) containing $100 \mu \mathrm{g} / \mathrm{ml}$ aprotinin, $2 \mathrm{mM} \mathrm{Na} \mathrm{NO}_{4}, 10 \mathrm{mM}$ $\mathrm{NaF}$, and protease inhibitor cocktail (Roche, Basel, Switzerland). Immunoblotting with the specific antibodies against COX-2 (Abcam, Cambridge, MA, USA), inducible nitric oxygen synthase (iNOS) (Abcam), $\beta$-actin (Sigma), inhibitor of NF-кB (ІкB)- $\alpha$ (Cell Signaling Technology, Beverly, MA, USA), or the phosphorylated form of IKB- $\alpha$ (Cell Signaling Technology) was performed using SDS-solubilized cellular proteins. Protein images were visualized by ImageQuant LAS-4000mini (Fujifilm, Tokyo, Japan) and quantified by Multi Gauge (Fujifilm).

\section{Measurement of lipid peroxide}

RAW 264.7 cells were incubated with or without $10 \%$ hemolysate treatment in the medium mixed with an equal part of saline, $10 \%$ trehalose, or $10 \%$ maltose for $3 \mathrm{~h}$. Collected cells were washed twice with PBS that was deoxidized by $\mathrm{N}_{2}$ aeration. The level of lipid peroxide (LPO) in the cells was measured using an LPO assay kit (Cayman) according to the manufacturer's instructions.

\section{Angiographic and histological examinations in the rabbit single-hemorrhage model}

The rabbit single-hemorrhage model was used as previously reported [24], with some modifications. Eighteen rabbits (weighing $2.8 \mathrm{~kg}$ to $3.4 \mathrm{~kg}$ ) were anesthetized with inhalation of isoflurane. Fresh autologous arterial blood was drawn from the ear artery. After $1.0 \mathrm{~mL}$ of cerebrospinal fluid was removed, $1.0 \mathrm{~mL}$ of blood was immediately injected into the cisterna magna with the animal in a tilted, head-down, prone position. The animal was maintained in the same position for 60 min to allow the injected blood to coagulate around the basilar artery. Three hours after the blood injection, the animal received an injection of $1.0 \mathrm{~mL}$ of saline, $30 \%$ trehalose, or $30 \%$ maltose into the cisterna magna in the Saline, Trehalose, or Maltose groups, respectively. Prior to and $48 \mathrm{~h}$ after the induction of experimental $\mathrm{SAH}$, a $4 \mathrm{~F}$ angiographic catheter was inserted into the left vertebral artery via the left femoral artery under fluoroscopy, and angiography of the basilar artery was performed by manual injection of $0.5 \mathrm{~mL}$ iohexol (350 mg of iodine/mL; Daiichi-Sankyo, Tokyo, Japan). After the angiography, mannitol $(0.5 \mathrm{mg} / \mathrm{kg} / \mathrm{h}$; Yoshindo, Toyama, Japan) was administered intravenously to prevent increased intracranial pressure.

Angiographic images of the basilar artery were adjusted with TOSPAC (Total System Performance Assessment Code; Toshiba, Tokyo, Japan). The diameter of the basilar 


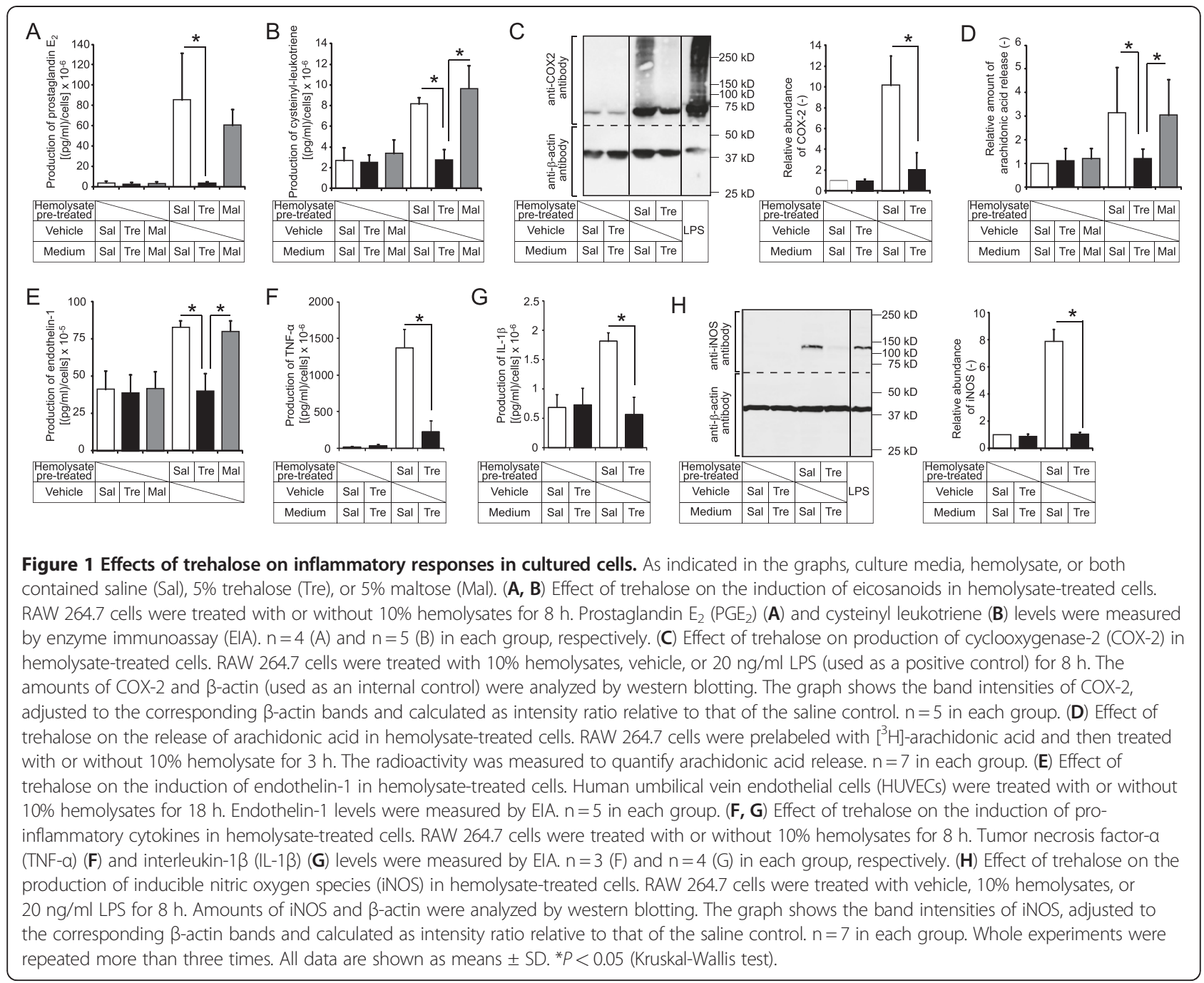

artery was measured as the mean diameters at three locations: distal, middle, and proximal. The length between the distal and proximal locations was set to 100 pixels on the computer monitor, and the middle location was set as 50 pixels from the distal and proximal locations. The real length per pixel was evaluated by measuring a $2 \mathrm{~mm}$ lead ball recorded at the same time as the basilar artery. These measurements were performed using a blinded method.

After perfusion fixation with $200 \mathrm{ml}$ of PBS and $450 \mathrm{ml}$ of $4 \%$ paraformaldehyde mixed with $50 \mathrm{ml}$ of $25 \%$ glutaraldehyde, the basilar arteries were sampled. Paraffin sections ( $3 \mu \mathrm{m}$ thick) of the basilar artery were prepared and then stained with hematoxylin and eosin (H\&E). The cross-sectional area of the vessel lumen was determined by measuring the circumference of the vessel lumen and calculating the area as a generalized circle. These measurements were also performed using a blinded method.
Rat femoral artery vasospasm model and immunostaining for cyclooxygenase-2, inducible nitric oxide synthase, and 7-ketocholesterol

We used the rat model for vasospasm established by Okada et al. [25], with modifications. After anesthesia with intraperitoneal injection of pentobarbital $(50 \mathrm{mg} / \mathrm{kg})$, both the left and right femoral arteries and veins in 60 rats (weighing 300 to $350 \mathrm{~g}$ ) were covered with a vinyl chloride catheter (10 $\mathrm{mm}$ in length). Fresh autologous blood was drawn from the femoral vein and mixed 3:1 with saline or $15 \%$ trehalose solution; the final concentration of trehalose was $3.75 \%$. The mixture was immediately injected into the strip of the left artery to fill it up. Saline alone was injected into the strip of the right artery as a control. At 1, 3, 5, 7, 10 , and 20 days after treatment, cryosections ( $3 \mu \mathrm{m}$ thick) of both femoral arteries were prepared and then stained with H\&E. The cross-sectional area of the vessel lumen was determined by measuring the circumference of the 
vessel lumen and calculating the area as a generalized circle. The wall media thickness was averaged among four distant sites. These measurements were performed using a blinded method. For each parameter, the ratio of the value in the left artery (tested artery) to that in the right artery (control artery) was calculated.

The cryosections were incubated overnight at $4^{\circ} \mathrm{C}$ with or without primary antibodies against cyclooxygenase-2 (COX-2) (1:500; Abcam), inducible nitric oxide synthase (iNOS) (1:500; Abcam), and 7-ketocholesterol (7-KC) (1:500; NOF, Tsukuba, Japan). Antibody localization was detected with HRP-conjugated secondary antibody (Promega, Madison, WI, USA) and diaminobenzidine (DAKO, Carpinteria, CA, USA). The sections were counterstained with methylgreen.

\section{Statistical analyses}

Data are expressed as means \pm SD or median and range, and were analyzed for significance by Kruskal-Wallis test with Bonferroni's correction or, when indicated, by Mann-Whitney's $U$ test. Values of $p<0.05$ were considered statistically significant.

\section{Results \\ Trehalose suppressed blood-induced inflammatory responses in vitro}

To confirm the effect of hemolysate on the arachidonic acid cascade, murine macrophage-like cells (RAW 264.7) were treated with $10 \%$ concentrations of hemolysate for various time periods. This treatment induced a significant time-dependent increase in the production of the eicosanoid $\mathrm{PGE}_{2}$ (Additional file 1: Figure S1A). Similar effects were observed in the production of cysteinyl leukotrienes (data not shown). To determine whether trehalose attenuated the production of $\mathrm{PGE}_{2}$, trehalose was mixed with hemolysate (pre-treatment), with the culture medium, or with both. Compared with the saline control, the increment in $\mathrm{PGE}_{2}$ production induced by hemolysate was significantly reduced by pre-treatment of the hemolysate with trehalose or by the presence of trehalose in the culture medium (Additional file 1: Figure S1B). While pretreatment was more effective than for culture medium treatment, the strongest effect was observed when the two were combined (Additional file 1: Figure S1B). By contrast, maltose, a structural isomer of trehalose with an $\alpha, \alpha-1,4$ glycosidic bond, had no effect on $\mathrm{PGE}_{2}$ production (Figure 1A). Similar results were obtained using HUVECs (Additional file 1: Figure S1C). Trehalose also suppressed the production of cysteinyl leukotrienes and prostaglandin $\mathrm{D}_{2}$, suggesting that trehalose treatment attenuates both COX and 5-lipoxygenase (5-LO) pathways, which metabolize arachidonic acid into prostaglandins and leukotrienes, respectively (Figure $1 \mathrm{~B}$ and Additional file 1: Figure S1D). Indeed, western blotting analysis revealed that the production of COX-2 (an inducible isoform of COX) elicited by hemolysate was significantly diminished by trehalose treatment in vitro (Figure 1C).

Next we evaluated the effect of trehalose on the action of cytosolic phospholipase $\mathrm{A}_{2} \mathrm{~S}\left(\mathrm{cPLA}_{2} \mathrm{~s}\right)$, which act upstream from COXs and 5-LO as rate-limiting enzymes in the arachidonic acid cascade, in hemolysate-treated cells. Treatment with hemolysate significantly increased the level of $\left[{ }^{3} \mathrm{H}\right]$-arachidonic acid release when saline or maltose was used (Figure 1D). Trehalose treatment suppressed hemolysate-induced $\left[{ }^{3} \mathrm{H}\right]$-arachidonic acid release (Figure 1D). To examine whether trehalose directly suppressed the enzyme activity of $\mathrm{CPLA}_{2}$, the phospholipid-cleaving activity of ${ }_{C P L A} \mathrm{~S}$ was evaluated in the presence of trehalose. The ${ }_{\mathrm{CPLA}} \mathrm{S}$ effectively cleaved the phospholipids in the presence or absence of trehalose (Additional file 1: Figure S1E), indicating that the reduction in lipid mediators by trehalose was not due to direct suppression of the enzyme activity of ${ }_{\mathrm{CPLA}} \mathrm{s}$. The production of endothelin- 1 , which is a potent vasoconstrictor and which was reported to be increased by treatment with hemolysate in cultured endothelial cells [26], was also suppressed by trehalose in HUVECs (Figure 1E).

Induction of various pro-inflammatory cytokines has been observed in both hemolysate-treated cells and experimental SAH models $[5,23,27]$. To investigate the effect of trehalose on cytokine expression, the levels of TNF- $\alpha$, IL- 6 , IL- $1 \alpha$, and IL- $1 \beta$ were measured in hemolysate-treated cultured cells. Trehalose significantly suppressed the hemolysate-induced productions of these cytokines (Figure 1F and G, and Additional file 1: Figure S1F and G). iNOS expression also plays an important role in the pathogenesis of cerebral injury after experimental SAH [20]. Thus, we examined the effect of trehalose on iNOS production induced by hemolysate treatment in cultured cells. Western blotting analysis showed that iNOS production was increased by hemolysate, but significantly reduced in trehalose-treated cells (Figure 1H).

Overall, these data suggest that trehalose inhibits the inflammatory responses induced by hemolysate in vitro.

\section{Trehalose suppressed activation of NF-KB induced by hemolysate in vitro}

$\mathrm{NF}-\mathrm{KB}$ is activated in the arterial wall after experimental $\mathrm{SAH}$, which may lead to induction of an inflammatory response including production of several cytokines [5]. We examined whether trehalose suppressed the activation of $\mathrm{NF}-\mathrm{KB}$ in hemolysate-treated macrophage cells in vitro. NF- $\mathrm{KB}$ is activated by the phosphorylation and resultant degradation of ІкB. Western blotting analysis showed that both the phosphorylation and degradation of ІкB- $\alpha$ were induced by hemolysate treatment (Figure 2A and B). Both of these effects were clearly suppressed by trehalose 


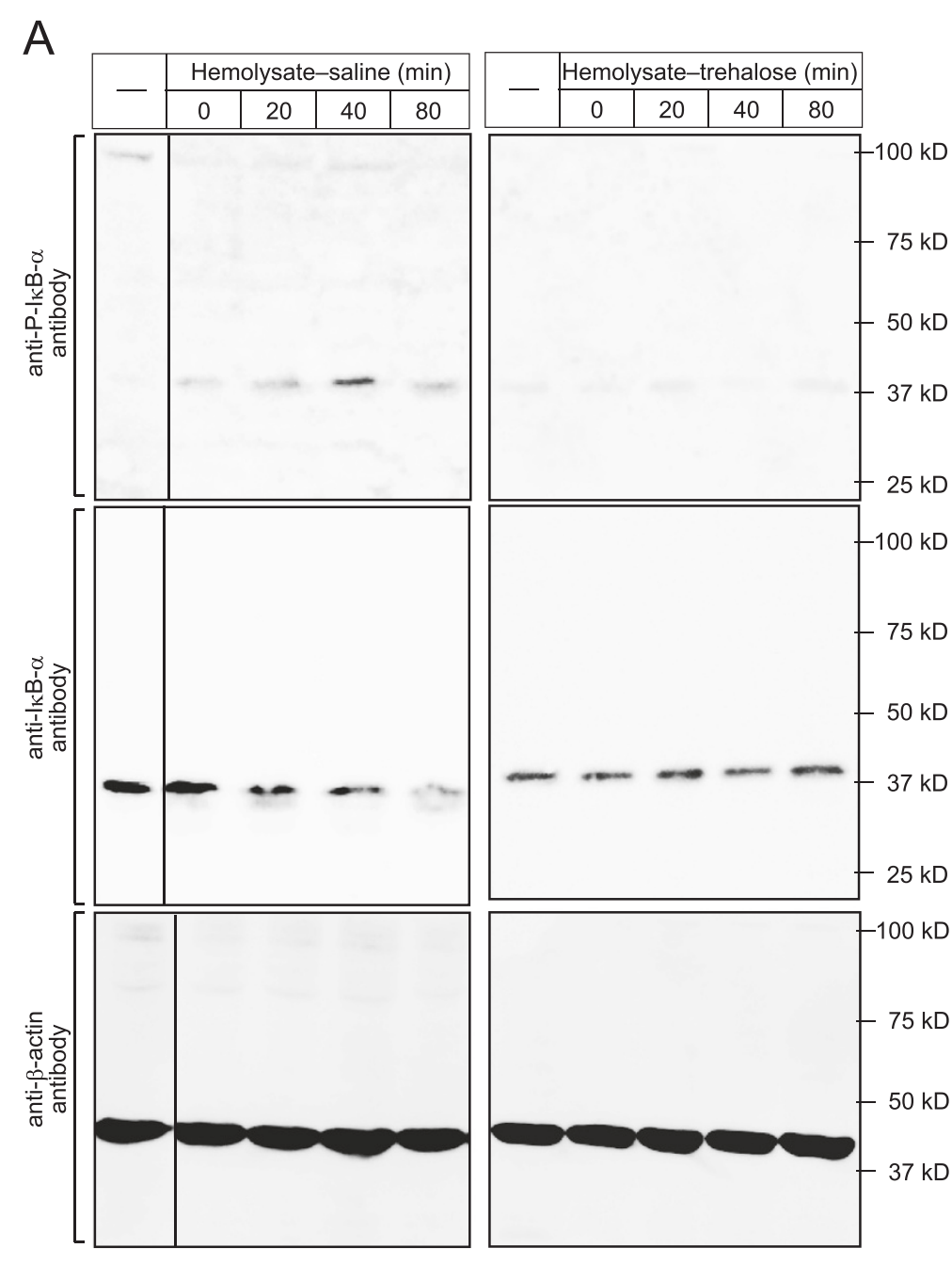

B

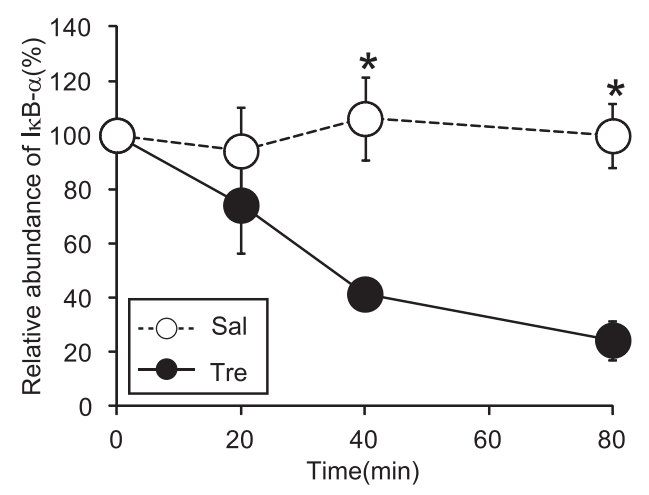

Figure 2 Effects of trehalose on the NF-KB pathway activated by hemolysate treatment. (A) Effect of trehalose on IkB-a phosphorylation and degradation in hemolysate-treated cells. RAW 264.7 cells were treated with 10\% hemolysates or vehicle for 80 min. Cells were collected at the indicated time points, and then SDS samples were prepared. Amounts of phosphorylated IKB-a (P-IKB-a), total IKB-a (IKB-a), and $\beta$-actin were analyzed by western blotting. (B) The graph shows changes in the band intensities of total IKB-a, adjusted to the corresponding $\beta$-actin bands and calculated as intensity ratio relative to that of 0 min time-point, for $0,20,40$, and 80 min. ${ }^{*} P<0.05$ as compared to saline (Mann-Whitney's $U$ test). $n=4$ in each group. Whole experiments were repeated more than three times. All data are means $\pm S D$. 
treatment in the hemolysate-treated cells (Figure 2A and B). These data suggest that trehalose suppresses the inflammatory responses induced by hemolysate via inhibition of the canonical NF-KB pathways.

\section{Trehalose protected against hemolysate- and ROS-induced oxidative stress in vitro}

Oxidative stress, including lipid peroxidation, has also been shown to contribute to cell injury and progression of early brain injury and cerebral vasospasm after SAH $[8,28]$. Oxyhemoglobin released from the lysis of red blood cells is considered to play a major role in oxidative cell damage via lipid peroxidation in and around the cerebral artery $[4,28]$. Furthermore, superoxide anion radicals and hydroxyl radicals, which are generated by and released from oxyhemoglobin, are involved in lipid peroxidation [4]. Thus, we examined whether trehalose directly suppressed the generation of LPO. Colorimetric analysis confirmed that lipid peroxidation was stimulated by treatment with hemolysate in cultured cells (Figure 3A). Hemolysate-induced lipid peroxidation was significantly suppressed by trehalose, but not by maltose (Figure 3A). Next, to examine the effect of trehalose on the oxidative stress-induced arachidonic acid release, we performed an arachidonic acid release assay primed by $\mathrm{H}_{2} \mathrm{O}_{2}$ in cultured cells. $\mathrm{H}_{2} \mathrm{O}_{2}$ was previously reported to induce arachidonic acid release via the lipid peroxidation $[29,30]$. In our assay, trehalose significantly suppressed $\mathrm{H}_{2} \mathrm{O}_{2}$-primed arachidonic acid release, while there was no effect of maltose (Figure 3B). These data suggest that trehalose directly reduces oxidative stress, including lipid peroxidation, induced by hemolysate and ROS.

To elucidate the mechanism by which trehalose suppressed oxidative stress, we investigated the effect of trehalose on the scavenging of free radicals using electron spin resonance spectroscopy with the spin-trap reagent 5(2,2-dimethyl-1,3-propoxy cyclophosphoryl)-5-methyl-1pyrroline N-oxide (CYPMPO) [31]. There was no effect of trehalose on the generation of super oxide anions using a hypoxanthine/xanthine oxidase system, whereas trehalose reduced the generation of hydroxyl radicals using a Fenton reaction, suggesting that trehalose scavenged hydroxyl radicals (Additional file 1: Figure S2). However, the scavenging effect of hydroxyl radicals was also observed with maltose (Additional file 1: Figure S2). Thus, these data suggest that the suppression mechanism of blood- and ROSinduced lipid peroxidation by trehalose is not primarily the result of the scavenging of free radicals.

\section{Trehalose suppressed cerebral vasospasm after the onset} of experimental SAH in the rabbit model

To test the effect of trehalose on cerebral vasospasm after experimental $\mathrm{SAH}$, we used the rabbit single- hemorrhage model. In preliminary experiments, blood mixed with saline, $3.8 \%$ trehalose, or $7.5 \%$ trehalose was administered into the cisterna magna of rabbits (coadministration model). Angiography revealed that the blood-induced vasospasm was weaker in the blood + trehalose group than in the saline group (Additional file 1: Figure S3). Trehalose at a concentration of $3.8 \%$ was less effective for the suppression of vasospasm than that of $7.5 \%$ (data not shown).

Next, to examine the effect of trehalose on cerebral vasospasm in a near-clinical setting, saline or trehalose at a final concentration of $7.5 \%$ was administered into the cisterna magna in a single dose at $3 \mathrm{~h}$ after blood injection (postadministration model). Blood in the cisterna magna was considered to be clotted at $3 \mathrm{~h}$ after blood injection. Similar to the case of co-administration of trehalose and blood, the vasospasm was significantly abrogated by postadministration of trehalose but not by post-administration of saline (Figure 4A and B). Maltose had a moderate inhibitory effect on vasospasm (Figure 4A and B). Histological analysis revealed that the characteristic features of vasospasm (reduction in the sectional area, thickening of the arterial wall, increase in endothelial cells, and corrugated internal elastic lamina) were observed in the basilar arteries of the saline- or maltose-injected animals, whereas the vasospasm was attenuated in the trehalose-injected animals (Figure 4C). Quantification of the cross-section of the basilar artery also revealed that post-injection of trehalose significantly suppressed the cerebral vasospasm (Figure 4D). Taken together, these data suggest that trehalose has a specific suppressive effect on the cerebral vasospasm caused by experimental $\mathrm{SAH}$, even when administered after the onset of experimental SAH.

Trehalose treatment abrogated blood-induced vasospasm, inflammatory responses, and lipid peroxidation in the presence of blood in the rat femoral artery model

In our rabbit model studies, it was not clear whether trehalose directly suppressed vasospasm or whether it facilitated the recovery from vasospasm in arterial tissue exposed to blood. To address this question, we performed the timecourse experiments using the rat femoral artery vasospasm model, which is analogous to clinical and experimental $\mathrm{SAH}$ in terms of the temporal evolution of vascular narrowing and morphological changes [25]. Control arteries exposed to saline for 7 days were similar in appearance to normal arteries (Figure 5B). Time-course experiments showed that the reduction in the sectional area and the thickening of the arterial walls both peaked at 7 days after treatment with a mixture of blood and saline (blood + saline), while these changes were suppressed by trehalose (final concentration of 3.75\%) (Figure 5A). On day 7, the characteristics of vasospasm were clearly observed in the arteries treated with blood + saline (Figure 5B). By contrast, 
A

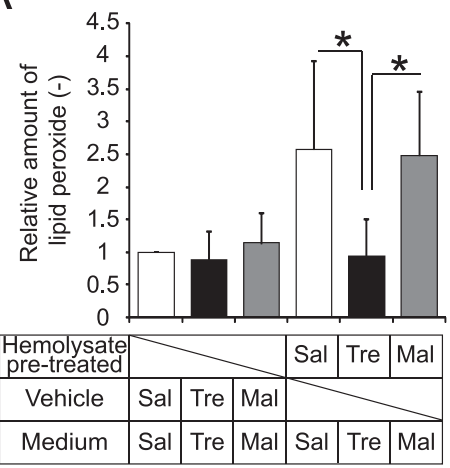

B

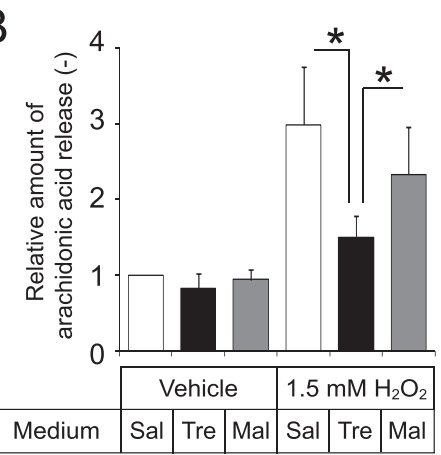

Figure 3 Effects of trehalose on hemolysate-induced and ROS-induced oxidative stress in cultured cells. As indicated in the graphs, the culture media, hemolysate, or both contained saline (Sal), 5\% trehalose (Tre), or 5\% maltose (Mal). (A) Measurement of lipid peroxide (LPO) using colorimetric analysis. RAW 264.7 cells were treated with or without 10\% hemolysates for 3 h. LPO levels were measured by a colorimetric assay using ferrous iron molecules. $\mathrm{n}=6$ in each group. (B) Effect of trehalose on the release of arachidonic acid in $\mathrm{H}_{2} \mathrm{O}_{2}$-treated cells. RAW 264.7 cells were prelabeled with $\left[{ }^{3} \mathrm{H}\right]$-arachidonic acid overnight and then treated with or without $1.5 \mathrm{mM} \mathrm{H}_{2} \mathrm{O}_{2}$ for $3 \mathrm{~h}$. The radioactivity of the collected culture media was measured to quantify arachidonic acid release. (Kruskal-Wallis test). $n=4$ in each group. All experiments were repeated more than three times. All data are shown as means $\pm \mathrm{SD}$. ${ }^{*} P<0.05$ (Kruskal-Wallis test).

the arteries exposed to blood + trehalose exhibited minimal changes (Figure 5B). Trehalose was also effective at final concentrations of $1.875 \%$ and $7.5 \%$ (Additional file 1: Figure
S4). These data suggest that trehalose directly prevented development of vasospasm induced by blood in the rat femoral artery model.

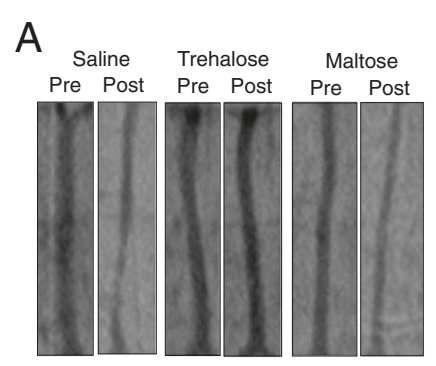

$\mathrm{B}$

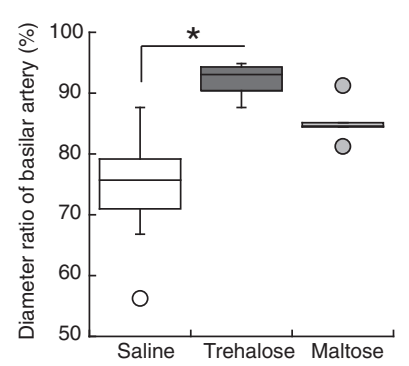

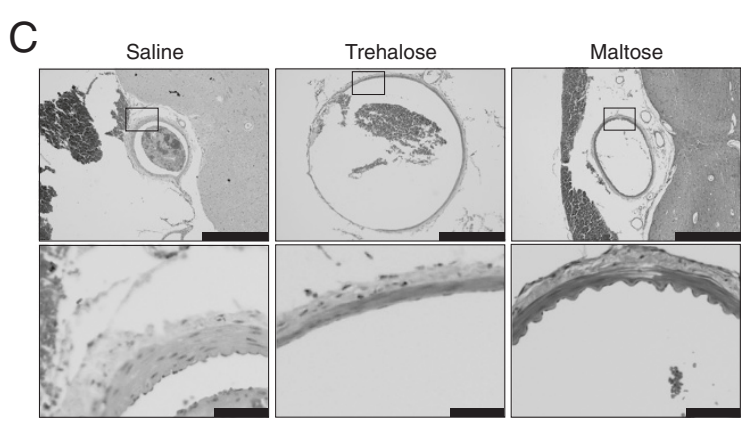

D

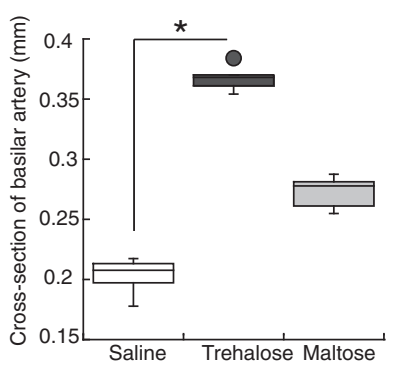

Figure 4 Effects of trehalose on cerebral vasospasm in experimental SAH (post-administration model). Saline, 30\% trehalose, or 30\% maltose was injected into the cisterna magna at $3 \mathrm{~h}$ after the experimental SAH induction. (A) Representative angiograms of the basilar arteries prior to (Pre) and $48 \mathrm{~h}$ after (Post) the induction of experimental SAH in rabbits. (B) The graph shows the diameter ratios of the basilar arteries after the induction of experimental SAH to the basilar arteries prior to the induction. ${ }^{*} P<0.05$ (Kruskal-Wallis test). $\mathrm{n}=6$ in each group. (C) Upper panels, representative H\&E-stained paraffin sections ( $3 \mu \mathrm{m}$ thick) of basilar arteries. Scale bars, $1000 \mu \mathrm{m}$. Lower panels, higher magnifications of the squares in the upper panels. Scale bars, $100 \mu \mathrm{m}$. (D) The graph shows the cross-section of basilar artery in each group. ${ }^{*} P<0.05$ (Kruskal-Wallis test). $n=6$ in each group. All experiments were repeated more than three times. The boxes in graphs indicate the 25th and 75 th quartiles and the central line is the median. The whiskers extend from the lowest to the highest value. Values outside the range of the whiskers are extreme values. 

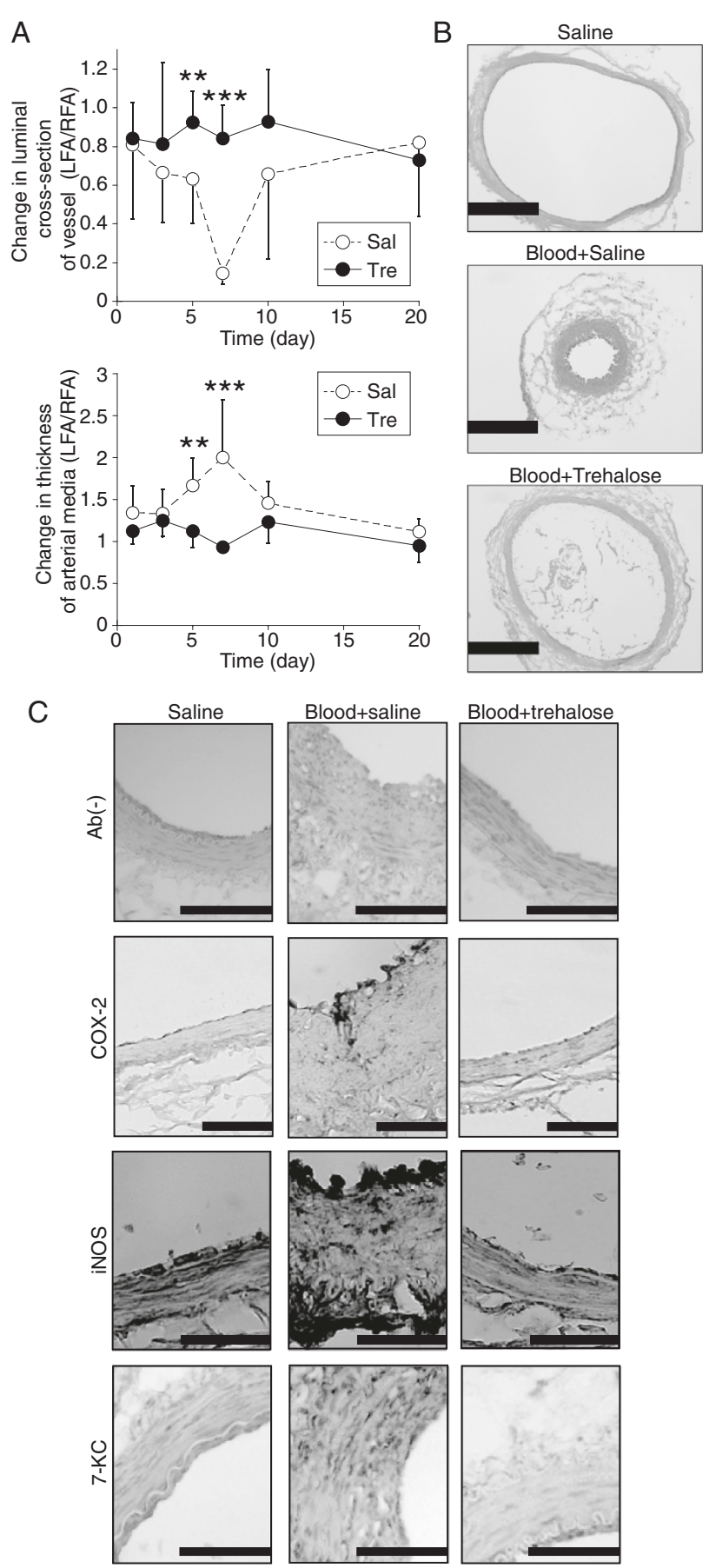

Figure 5 Effects of trehalose on inflammatory responses, oxidative stress, and vasospasm in the presence of blood. (A) Changes in the luminal cross-sectional area and thickness of the arterial media of femoral vessels exposed to saline or to blood containing either saline or trehalose for 1, 3, 5, 7, 10, and 20 days. The ratios of the luminal cross-sectional area and the thickness of the arterial media of femoral vessels exposed to blood containing either saline (Sal) or 3.75\% trehalose (Tre) to those exposed to saline alone were calculated. ${ }^{* *} P<0.01$ and ${ }^{* * *} P<0.005 *$ as compared with saline (Mann-Whitney's $U$ test). $n=6$ in each group. (B) Representative H\&E-stained cryosections ( $3 \mu m$ thick) of femoral arteries exposed to saline alone (Saline) or to blood containing either saline (Blood + Saline) or 3.75\% trehalose (Blood + Trehalose) at 7 days. Scale bars, $200 \mu \mathrm{m}$. (C) Immunohistological analysis in the rat artery vasospasm model. The cryosections (3 $\mu \mathrm{m}$ thick) were immunostained with or without antibodies (Ab) against COX-2, iNOS, or 7-ketocholesterol (KC) and then counterstained with methyl green. Scale bars, $20 \mu \mathrm{m}$. All experiments were repeated more than three times. All data are shown as means \pm SD. 
Next, we examined whether trehalose suppressed the activation of inflammatory responses and production of lipid peroxide induced by blood. We performed immunohistochemical analysis for the inflammatory markers COX-2 and iNOS in the rat models. In the blood + saline groups, endothelial cells in the intimal layer were stained with the COX-2 antibody, and endothelial cells in the intimal layer and fibroblasts in the adventitial layer were stained with the iNOS antibody (Figure 5C). By contrast, the immunostaining intensities for both COX-2 and iNOS were markedly diminished in the blood + trehalose groups and the negative control groups (Figure 5C). Immunohistochemical analysis also detected 7-KC, a marker for lipid peroxidation and a non-enzymatic oxidant of cholesterol [29], in smooth muscle cells of medial layer in the blood + saline group. By contrast, $7-\mathrm{KC}$ production was not observed in the blood + trehalose group (Figure 5C). These data suggest that trehalose suppresses inflammatory responses and lipid peroxidation as well as the induction of vasospasm in vivo.

\section{Discussion}

In the present study, we demonstrate that (1) trehalose suppressed hemolysate-induced inflammatory responses including activation of the arachidonic acid cascade and production of iNOS, pro-inflammatory cytokines, and endothelin-1 in cultured cells; (2) trehalose suppressed activation of the NF-kB pathway in the hemolysate-treated cultured cells; (3) trehalose protected cells from hemolysateand ROS-induced lipid peroxidation in the cultured cells; (4) a single administration of trehalose after the onset of experimental SAH suppressed blood-induced vasospasm in the rabbit single-hemorrhage model; and (5) trehalose suppressed development of vasospasm, as well as inflammatory responses and lipid peroxidation in the presence of blood in the rat femoral artery model.

\section{Putative mechanisms of suppression of vasospasm by trehalose in subarachnoid space after the onset of experimental SAH}

Our rabbit model studies showed that a single administration of trehalose even after the onset of experimental SAH suppressed cerebral vasospasm. In addition, we observed that trehalose suppressed development of vasospasm even in the presence of blood in the rat femoral artery model. These results suggest that trehalose, by remaining in the subarachnoid space, can suppress complications of SAH even if the blood clots are remained. It is unclear how trehalose exerts this anti-vasospasm effect on arterial tissue. Nevertheless, autoradioluminogram of the rabbits after intraperitoneal administration of radiolabeled-trehalose revealed that trehalose remained in intraperitoneal cavity and was relatively localized on the organ surface (likely through interaction with membrane) for several hours, while the trehalose was then transported to various other organs within $24 \mathrm{~h}$ (S. Kuribayashi, Personal communication). Therefore, similarly in the intraperitoneal space, trehalose may remain in the subarachnoid space for several hours and suppress cerebral vasospasm through its membrane-protective effect.

\section{Putative mechanisms of the anti-inflammatory effect of trehalose after treatment with blood}

A number of reports have suggested an association between inflammatory responses and complications such as cerebral vasospasm after SAH $[4,27,32]$. We observed that hemolysate induced the production of several inflammatory mediators as well as the activation of NF-кB in vitro. We also confirmed that COX-2 and iNOS were produced in the rat femoral artery model, similar to the results with experimental SAH models previously described $[19,20]$. Our in vivo and in vitro studies clearly showed trehalose suppressed these inflammatory responses.

Production of the lipid mediators is mainly controlled by $\mathrm{CPLA}_{2}$. However, trehalose did not directly inhibit the enzyme activity of cPLA ${ }_{2}$, despite a decrease in production of lipid mediators and arachidonic acid release induced by hemolysate treatment in cultured cells. The $\mathrm{Ca}^{2+}$-independent $\mathrm{PLA}_{2} \quad\left(\mathrm{PLA}_{2}\right.$, group VI $\mathrm{PLA}_{2}$ ) is involved in arachidonic acid release induced by various stimulations and nicotine-induced contraction in the basilar artery via arachidonic acid metabolites [33,34]. Thus, iPLA $_{2}$ may be involved in arachidonic acid release and vasospasm induced by blood, and trehalose may suppress the enzyme activity of PLA $_{2}$.

Production of pro-inflammatory mediators is partially regulated by the NF-кB pathway [35-37]. Our findings, in combination with those previously reported, suggest that trehalose suppresses the blood-induced inflammatory responses by inhibiting the NF- $\mathrm{KB}$ pathway. In support of these data, previous studies have demonstrated that trehalose prevented pro-inflammatory activation by endotoxic shock both in vitro and in vivo, potentially through several signaling pathways including the NF-кB pathway $[13,14]$. Thus, trehalose may be useful as a novel anti-inflammatory therapy.

We observed that pre-treatment of the hemolysate with trehalose was more effective than the presence of trehalose in culture medium for relieving blood-induced lipid mediator production in vitro. Sphingosylphosphorylcholine was recently identified as a pro-inflammatory factor after experimental SAH [38]. The anti-inflammatory effect of trehalose may be derived, at least in part, from inactivation of such pro-inflammatory factor (s). We also consider that the anti-inflammatory actions of trehalose are regulated by an identified or unidentified trehalose receptor expressed on the cell surface, and that this receptor attenuates activation of the signal transduction cascade such as the arachidonic 
acid cascade and the NF-кB pathway. Indeed, Taya et al. suggested that LPS-induced cytokine productions might be inhibited by trehalose via the T1R3 trehalose receptor in macrophage cells [39].

\section{Putative mechanisms of the antioxidant effect of trehalose after treatment with blood}

The development of cerebral injury after SAH is triggered by oxidative stress including lipid peroxidation of the cell membrane [7]. Herein, we showed that trehalose suppressed the generation of lipid peroxidation induced by blood. Consistent with these data, trehalose was previously reported to suppress ROS-induced lipid peroxidation in yeast cells [40]. Trehalose may suppress lipid peroxidation by directly interacting with the membrane and thereby suppressing radical oxidation of unsaturated fatty acids [41]. Although trehalose appears to have an effect similar to free radical scavengers, the mechanism of action of trehalose clearly differs from that of free radical scavengers in that it protects the cell membrane.

\section{The effect of maltose on vasospasm, inflammation, and lipid peroxidation}

While maltose had a moderate effect on vasospasm in vivo unlike trehalose, it was not effective in reducing bloodinduced inflammation and lipid peroxidation. These results are in accordance with a previous report showing that maltose had no effect on an endotoxin-induced proinflammatory phenotype [14]. It is likely that the antiinflammatory and antioxidant effects of trehalose are derived from its unique structural properties, not observed in maltose, that strongly interact with proteins and lipids through hydrogen bonding [42]. Further studies are required to determine the effect of trehalose on various pro-inflammatory factors and oxidants.

\section{Possible clinical use of trehalose after SAH}

The most well established method for preventing the complications of SAH includes cisternal irrigation therapy with urokinase and intracisternal administration of tissue plasminogen activator. We found that injection of trehalose into the subarachnoid space after the onset of experimental $\mathrm{SAH}$ was able to inhibit vasospasm. Thus, in the clinical setting, we suggest that cisternal irrigation fluid is the optimal route of trehalose administration, and is anticipated to have an additional or synergistic therapeutic effect on complications of $\mathrm{SAH}$ when combined with existing therapeutic modalities.

\section{Study limitations}

There are some limitations in this study. First, while we showed suppressive effects of trehalose on inflammation, oxidative stress, and vasospasm, we could not fully elucidate the relationship between these pathological mechanisms and the effects of trehalose. Secondly, although we consider that trehalose could be administered with cisternal irrigation therapy in the clinical setting, we examined the effect of trehalose on vasospasm via a bolus injection into the cisternal space, not cisternal irrigation. Thirdly, we only examined the inflammatory response and oxidative stress in the femoral arterial walls only. Finally, we did not evaluate any cerebral function and morphology such as mortality, neurological function, cerebral blood flow, and neuronal cell death in the rabbit model.

\section{Conclusions}

We demonstrated that periadventitial treatment with trehalose can produce pleiotropic actions including inhibition of inflammatory responses, lipid peroxidation, and vasospasm. The presence of these multiple effects is advantageous compared with other drug candidates such as radical scavengers or anti-inflammatory reagents that exert only a single action. Trehalose has already been used as an organ preservation solution in clinical transplantation, and has no side effects. Thus, trehalose may be a potential therapeutic option for patients with SAH, either as monotherapy or in combination with other treatment modalities.

\section{Additional file}

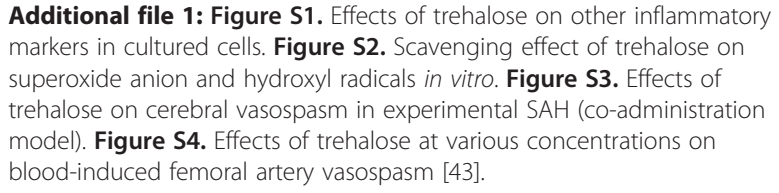

Additional file 1: Figure S1. Effects of trehalose on other inflammatory markers in cultured cells. Figure S2. Scavenging effect of trehalose on superoxide anion and hydroxyl radicals in vitro. Figure S3. Effects of trehalose on cerebral vasospasm in experimental SAH (co-administration model). Figure S4. Effects of trehalose at various concentrations on blood-induced femoral artery vasospasm [43]

\section{Competing interests}

The authors declare that they have no competing interests.

\section{Acknowledgements}

We thank Takayoshi Wakagi for technical advice on ESR, Toru Egashira for technical advice on the analysis of lipid peroxidation results, and Toru Hakukawa, Hiroshi Ueno, and Yukako Suzuki for their technical assistance. The present work was supported by grants from the Terumo Life Science Foundation and by a Grant-in-Aid for Challenging Exploratory Research from the Japanese Ministry of Education, Culture, Sports, Science, and Technology (Project No. 21650105), the Center for NanoBio Integration (CNBI program), the Center for Medical System Innovation (CMSI program), and the Funding Program for World-Leading Innovative R\&D on Science and Technology (FIRST program)

\section{Author details}

${ }^{1}$ Laboratory of Veterinary Surgery, Graduate School of Agricultural and Life Sciences, The University of Tokyo, 1-1-1 Yayoi, Bunkyo-ku, Tokyo 113-8657, Japan. ${ }^{2}$ NEXT21 K.K., 3-38-1 Hongo, Bunkyo-ku, Tokyo 113-0033, Japan. ${ }^{3}$ Center for Disease Biology and Integrative Medicine, Faculty of Medicine, The University of Tokyo, 7-3-1 Hongo, Bunkyo-ku, Tokyo 113-0033, Japan. ${ }^{4}$ Department of Bioengineering, Graduate School of Engineering, The University of Tokyo, 2-11-16 Yayoi, Bunkyo-ku, Tokyo 113-8656, Japan. ${ }^{5}$ Department of Biochemistry and Molecular Biology, Faculty of Medicine, The University of Tokyo, 7-3-1 Hongo, Bunkyo-ku, Tokyo 113-0033, Japan.

\section{Authors' contributions}

RE participated in the design of the study, performed in vivo experiments using rabbits and rats, and drafted the manuscript. NS participated in the 
design of the study, performed in vitro experiments, and drafted the manuscript. KK partly performed in vivo experiments using rabbits. FY performed immunohistological experiments. YK-K partly performed in vivo experiments using rats. TO performed in vitro PLA 2 assay. AF, YK, MN, SS, MM, TS, UC, and NS participated in the study design and coordination and drafted the manuscript. All authors read and approved the final manuscript.

Received: 15 December 2011 Accepted: 30 April 2012

Published: 30 April 2012

\section{References}

1. van Gijn J, Kerr RS, Rinkel GJ: Subarachnoid haemorrhage. Lancet 2007, 369:306-318.

2. Macdonald RL, Kassell NF, Mayer S, Ruefenacht D, Schmiedek P, Weidauer S, Frey A, Roux S, Pasqualin A, Investigators C: Clazosentan to Overcome Neurological Ischemia and Infarction Occurring After Subarachnoid Hemorrhage (CONSCIOUS-1) randomized, double-blind, placebocontrolled phase 2 dose-finding trial. Stroke 2008, 39:3015-3021.

3. Macdonald RL, Pluta RM, Zhang JH: Cerebral vasospasm after subarachnoid hemorrhage: the emerging revolution. Nat Clin Pract Neurol 2007, 3:256-263.

4. Sercombe R, Dinh YR, Gomis P: Cerebrovascular inflammation following subarachnoid hemorrhage. Jpn J Pharmacol 2002, 88:227-249.

5. Zhou M-L, Shi J-X, Hang C-H, Cheng H-L, Qi X-P, Mao L, Chen K-F, Yin H-X Potential contribution of nuclear factor-KB to cerebral vasospasm after experimental subarachnoid hemorrhage in rabbits. J Cereb Blood Flow Metab 2007, 27:1583-1592.

6. Rodriguez y Baena R, Gaetani P, Folco G, Vigano T, Paoletti P: Arachidonate metabolites and vasospasm after subarachnoid haemorrhage. Neurol Res 1986, 8:25-32.

7. Ayer RE, Zhang JH: Oxidative stress in subarachnoid haemorrhage: significance in acute brain injury and vasospasm. Acta Neurochir Supp/ 2008, 104:33-41

8. Endo H, Nito C, Kamada H, Yu F, Chan PH: Reduction in oxidative stress by superoxide dismutase overexpression attenuates acute brain injury after subarachnoid hemorrhage via activation of Akt/glycogen synthase kinase-3 $\beta$ survival signaling. J Cereb Blood Flow Metab 2007, 27:975-982.

9. Komotar RJ, Zacharia BE, Valhora R, Mocco J, Connolly ES Jr: Advances in vasospasm treatment and prevention. J Neurol Sci 2007, 261:134-142.

10. Al-Tamimi YZ, Orsi NM, Quinn AC, Homer-Vanniasinkam S, Ross SA: A review of delayed ischemic neurologic deficit following aneurysmal subarachnoid hemorrhage: historical overview, current treatment, and pathophysiology. World Neurosurg 2010, 73:654-667.

11. Elbein AD, Pan YT, Pastuszak I, Carroll D: New insights on trehalose: a multifunctional molecule. Glycobiology 2003, 13:17R-27R.

12. Jain NK, Roy I: Effect of trehalose on protein structure. Protein Sci 2009, 18:24-36.

13. Minutoli L, Altavilla D, Bitto A, Polito F, Bellocco E, Lagana G, Fiumara T, Magazu S, Migliardo F, Venuti FS, Squadrito F: Trehalose: a biophysics approach to modulate the inflammatory response during endotoxic shock. Eur J Pharmacol 2008, 589:272-280.

14. Minutoli L, Altavilla D, Bitto A, Polito F, Bellocco E, Lagana G, Giuliani D, Fiumara T, Magazu S, Ruggeri $P$, et al: The disaccharide trehalose inhibits proinflammatory phenotype activation in macrophages and prevents mortality in experimental septic shock. Shock 2007, 27:91-96.

15. Chen F, Fukuse T, Hasegawa S, Bando T, Hanaoka N, Kawashima M, Sakai H, Hamakawa H, Fujinaga T, Nakamura T, Wada H: Effective application of ETKyoto solution for clinical lung transplantation. Transplant Proc 2004 36:2812-2815

16. Tablin F, Wolkers WF, Walker NJ, Crowe JH: Treahlose loaded, freeze-dried human platelets are functional and retain normal protein structure and membrane phospholipid phase transitions. Blood 2000, 96:62a-62a.

17. Matsuo T, Tsuchida Y, Morimoto N: Trehalose eye drops in the treatment of dry eye syndrome. Ophthalmology 2002, 109:2024-2029.

18. Mori Y, Yano F, Shimohata N, Suzuki S, Chung UI, Takato T: Trehalose inhibits oral dryness by protecting the cell membrane. Int J Oral Maxillofac Surg 2010, 39:916-921.

19. Tran Dinh YR, Jomaa A, Callebert J, Reynier-Rebuffel AM, Tedgui A, Savarit A, Sercombe R: Overexpression of cyclooxygenase- 2 in rabbit basilar artery endothelial cells after subarachnoid hemorrhage. Neurosurgery 2001, 48:626-633; discussion 633-635.

20. Widenka DC, Medele RJ, Stummer W, Bise K, Steiger HJ: Inducible nitric oxide synthase: a possible key factor in the pathogenesis of chronic vasospasm after experimental subarachnoid hemorrhage. J Neurosurg 1999, 90:1098-1104.

21. Oshiro EM, Hoffman PA, Dietsch GN, Watts MC, Pardoll DM, Tamargo RJ: Inhibition of experimental vasospasm with anti-intercellular adhesion molecule-1 monoclonal antibody in rats. Stroke 1997, 28:2031-2037; discussion 2037-2038.

22. Thai QA, Oshiro EM, Tamargo RJ: Inhibition of experimental vasospasm in rats with the periadventitial administration of ibuprofen using controlled-release polymers. Stroke 1999, 30:140-147.

23. Sasaki T, Kasuya H, Onda H, Sasahara A, Goto S, Hori T, Inoue I: Role of p38 mitogen-activated protein kinase on cerebral vasospasm after subarachnoid hemorrhage. Stroke 2004, 35:1466-1470.

24. Tang WH, Chen Z, Liu Z, Zhang JH, Xi G, Feng H: The effect of ecdysterone on cerebral vasospasm following experimental subarachnoid hemorrhage in vitro and in vivo. Neurol Res 2008, 30:571-580.

25. Okada T, Harada T, Bark DH, Mayberg MR: A rat femoral artery model for vasospasm. Neurosurgery 1990, 27:349-356.

26. Chang $C Z$, Winardi $D$, Lin $C L$, Kwan AL, Jeng AY, Kassell NF, Howng SL, Lee KS: Attenuation of hemolysate-induced cerebrovascular endothelial cell injury and of production of endothelin- 1 and big endothelin-1 by an endothelin-converting enzyme inhibitor. Surg Neurol 2002, 58:181-187. discussion 187-188.

27. Dumont AS, Dumont RJ, Chow MM, Lin C-I, Calisaneller T, Ley KF, Kassell NF, Lee KS: Cerebral Vasospasm after Subarachnoid Hemorrhage: Putative Role of Inflammation. Neurosurgery 2003, 53:123-135.

28. Asano T, Matsui T: Antioxidant therapy against cerebral vasospasm following aneurysmal subarachnoid hemorrhage. Cell Mol Neurobiol 1999, 19:31-44.

29. Deiana M, Incani A, Rosa A, Corona G, Atzeri A, Loru D, Paola Melis M, Assunta Dessi M: Protective effect of hydroxytyrosol and its metabolite homovanillic alcohol on $\mathrm{H}_{2} \mathrm{O}_{2}$ induced lipid peroxidation in renal tubular epithelial cells. Food Chem Toxicol 2008, 46:2984-2990.

30. Han WK, Sapirstein A, Hung CC, Alessandrini A, Bonventre JV: Cross-talk between cytosolic phospholipase $A_{2}$ a $\left(C P L A_{2} a\right)$ and secretory phospholipase $A_{2}\left(S P L A_{2}\right)$ in hydrogen peroxide-induced arachidonic acid release in murine mesangial cells: $\mathrm{SPLA}_{2}$ regulates $\mathrm{CPLA}_{2} \mathrm{a}$ activity that is responsible for arachidonic acid release. J Biol Chem 2003, 278:24153-24163.

31. Kamibayashi M, Oowada S, Kameda H, Okada T, Inanami O, Ohta S, Ozawa T, Makino K, Kotake Y: Synthesis and characterization of a practically better DEPMPO-type spin trap, 5-(2,2-dimethyl-1,3-propoxy cyclophosphoryl)-5methyl-1-pyrroline N-oxide (CYPMPO). Free Radic Res 2006, 40:1166-1172.

32. Macdonald RL, Weir BK: A review of hemoglobin and the pathogenesis of cerebral vasospasm. Stroke 1991, 22:971-982.

33. Ji X, Nishihashi T, Trandafir CC, Wang A, Shimizu Y, Kurahashi K: Pharmacological nature of nicotine-induced contraction in the rat basilar artery: involvement of arachidonic acid metabolites. Eur J Pharmacol 2007, 577:109-114.

34. Akiba S, Mizunaga S, Kume K, Hayama M, Sato T: Involvement of group VI $\mathrm{Ca}^{2}{ }^{+}$- independent phospholipase $\mathrm{A}_{2}$ in protein kinase $\mathrm{C}$-dependent arachidonic acid liberation in zymosan-stimulated macrophage-like P388D 1 cells. J Biol Chem 1999, 274:19906-19912.

35. Tak PP, Firestein GS: NF-kB: a key role in inflammatory diseases. J Clin Invest 2001, 107:7-11

36. Kyriakis JM, Avruch J: Mammalian mitogen-activated protein kinase signal transduction pathways activated by stress and inflammation. Physiol Rev 2001, 81:807-869.

37. Hirabayashi T, Murayama T, Shimizu T: Regulatory mechanism and physiological role of cytosolic phospholipase A2. Biol Pharm Bull 2004, 27:1168-1173.

38. Wirrig C, Hunter I, Mathieson FA, Nixon GF: Sphingosylphosphorylcholine is a proinflammatory mediator in cerebral arteries. J Cereb Blood Flow Metab 2011, 31:212-221.

39. Taya K, Hirose K, Hamada S: Trehalose inhibits inflammatory cytokine production by protecting IKB-a reduction in mouse peritoneal macrophages. Arch Oral Biol 2009, 54:749-756. 
40. Herdeiro RS, Pereira MD, Panek AD, Eleutherio EC: Trehalose protects

Saccharomyces cerevisiae from lipid peroxidation during oxidative stress. Biochim Biophys Acta 2006, 1760:340-346.

41. Oku K, Watanabe H, Kubota M, Fukuda S, Kurimoto M, Tsujisaka Y, Komori M, Inoue $Y$, Sakurai M: NMR and quantum chemical study on the $\mathrm{OH}$....pi and $\mathrm{CH}$. . . O interactions between trehalose and unsaturated fatty acids: implication for the mechanism of antioxidant function of trehalose. J Am Chem Soc 2003, 125:12739-12748.

42. Crowe JH, Carpenter JF, Crowe LM: The role of vitrification in anhydrobiosis. Annu Rev Physiol 1998, 60:73-103.

43. Ohto $T$, Uozumi $N$, Hirabayashi $T$, Shimizu T: Identification of novel cytosolic phospholipase $A_{2} s$, murine $C P L A_{2} \delta$, and $\zeta$, which form a gene cluster with $\mathrm{CPLA}_{2} \beta$. J Biol Chem 2005, 280:24576-24583.

doi:10.1186/1479-5876-10-80

Cite this article as: Echigo et al:: Trehalose treatment suppresses

inflammation, oxidative stress, and vasospasm induced by experimental

subarachnoid hemorrhage. Journal of Translational Medicine 2012 10:80.

\section{Submit your next manuscript to BioMed Central and take full advantage of:}

- Convenient online submission

- Thorough peer review

- No space constraints or color figure charges

- Immediate publication on acceptance

- Inclusion in PubMed, CAS, Scopus and Google Scholar

- Research which is freely available for redistribution 